\title{
Coherent risk measures, coherent capital allocations, and the gradient allocation principle
}

\author{
ARNE BUCH*, GREGOR DORFLEITNER* \\ * Institute of Finance and Financial Markets, Vienna University of Economics and Business Administration
}

Summary The gradient allocation principle, which generalizes the most popular specific allocation principles, is commonly proposed in the literature as a means of distributing a financial institution's risk capital to its constituents. This paper is concerned with the axioms defining the coherence of risk measures and capital allocations, and establishes results linking the two coherence concepts in the context of the gradient allocation principle. The following axiom pairs are shown to be equivalent: positive homogeneity and full allocation, subadditivity and "no undercut", and translation invariance and riskless allocation. Furthermore, we point out that the symmetry property holds if and only if the risk measure is linear. As a consequence, the gradient allocation principle associated with a coherent risk measure has the properties of full allocation and "no undercut", but not symmetry unless the risk measure is linear. The results of this paper are applied to the covariance, the semi-covariance, and the expected shortfall principles. We find that the gradient allocation principle associated with a non-linear risk measure can be coherent, in a suitably restricted setting.

Keywords: Risk capital allocation; Gradient allocation principle; Coherent risk measures; Coherent capital allocations 


\section{Introduction}

Regulatory requirements and the need to maintain a certain safety level are two reasons for financial institutions to retain risk capital. The amount held on the corporate level can be determined using an appropriate risk measure. In Artzner et al. (1999), four desirable axioms defining the coherence of a risk measure are given and justified. These axioms are translation invariance, subadditivity, positive homogeneity, and monotonicity. The sum of the risk capitals of the constituents standing alone typically exceeds the total risk capital of the firm due to diversification effects. These need to be fairly accounted for when the overall buffer is distributed to business units or single contracts, for the purpose of performance measurement or premium calculations.

In the widely recognized paper of Denault (2001), three desirable axioms defining the coherence of allocation principles are introduced. These principles are "no undercut", symmetry, and riskless allocation, and taken together motivate the gradient allocation principle. After identifying risk measures and cost functions, coherent allocation principles and coherent fuzzy values, and applying the theory of coalitional games with fractional players, it is shown that the gradient of a differentiable cost function specifies a coherent fuzzy value. The gradient allocation principle also arises from the axioms proposed in Kalkbrener (2005). A further justification for the gradient allocation principle is provided by Tasche (2000), where it is shown that the gradient of a differentiable risk measure with continuous derivatives is the unique continuous per-unit allocation principle suitable for performance measurement on the basis of a risk-adjusted return function.

This paper focuses on the risk measure and allocation principle coherence concepts as applied to the gradient allocation principle. It is shown that three of the coherent risk measure axioms are sufficient for two of the coherent allocation principle axioms. The implications of the coherent allocation principle axioms on the risk measure are also presented. The results of this analysis establish the equivalence of positive homogeneity and full allocation, of subadditivity and "no undercut", and of translation invariance and riskless allocation. It is further shown that the symmetry property holds if and only if the risk measure is linear, and hence does not account for diversification effects. Consequently, the gradient principle with a coherent risk measure satisfies the full allocation and "no undercut" axioms, but not the symmetry axiom unless the risk measure is linear. Furthermore, we find that the gradient allocation principle associated with a risk measure based on expectation and standard deviation can be coherent in a restricted setting.

This paper is organized as follows. In section 2 the notion of coherent risk measures is recalled, and a general definition of coherent capital allocations is given allowing for the insight by Fischer (2003) that differentiability of a positively homogeneous and subadditive risk measure on all portfolios corresponds to linearity of the risk measure. In section 3, the consequences of the coherency axioms are stated. In section 4, the results are applied 
to certain popular risk measures based on standard deviation, semi-covariance and expected shortfall and the corresponding covariance, semi-covariance, and expected shortfall allocation principles. Moreover, it is shown that the covariance principle is coherent when restricted to centered random variables. Concluding remarks concerning economical implications end this paper.

\section{The notions of coherent risk measure and of coherent capital allocation}

We follow Artzner et al. (1999) in understanding the risk of a position or firm to be related to the variability of its net worth over the next period. The future worth is represented by the set $\mathfrak{X}$ of bounded random variables on the probability space $[\Omega, \mathfrak{A}, \mathbb{P}]$. The risk associated with $X \in \mathfrak{X}$ is quantified by a risk measure $\rho$; formally, this measure is a mapping of $\mathfrak{X}$ into the real numbers $\mathbb{R}$. Note that the assumption of bounded random variables is simply made for convenience. For a given risk measure, all the results stated in this paper can be generalized to the set of random variables whose every element entails a well-defined risk.

Artzner et al. have introduced (1997) and further developed (1999) an axiomatic characterization known as "the concept of coherent risk measures", which is defined as follows. For the sake of simplicity, the interest rate $r$ is assumed to be equal to 1 throughout this paper.

Definition 2.1 A risk measure $\rho$ satisfying the following four properties is called coherent:

1. Translation invariance: for all $X \in \mathfrak{X}$ and all real numbers $\alpha$, we have $\rho(X+\alpha)=\rho(X)-\alpha$.

2. Subadditivity: for all $X_{1}$ and $X_{2} \in \mathfrak{X}$, we have $\rho\left(X_{1}+X_{2}\right) \leq \rho\left(X_{1}\right)+\rho\left(X_{2}\right)$.

3. Positive homogeneity: for all $\lambda>0$ and all $X \in \mathfrak{X}, \rho(\lambda X)=\lambda \rho(X)$.

4. Monotonicity: for all $X$ and $Y \in \mathfrak{X}$ with $X \leq Y$, we have $\rho(Y) \leq \rho(X)$.

Note that a positively homogeneous and subadditive functional can be referred to as sublinear. The following relations hold under the axioms of translation invariance and positive homogeneity: $\rho(0)-\alpha=\rho(0+\alpha)=$ $\rho(0+\alpha) / 2+\rho(0+\alpha) / 2=\rho(0+\alpha / 2)+\rho(0+\alpha / 2)=2 \cdot \rho(0)-\alpha$; the beginning and end of this chain imply that $\rho(0)=0$. Hence, Definition 2.1 is equivalent to the original version presented by Artzner et al., where positive homogeneity is stated as follows: for all $\lambda \geq 0$ and all $X \in \mathfrak{X}, \rho(\lambda X)=\lambda \rho(X)$.

For an economic interpretation of these four axioms, we refer the reader to Artzner et al. (1999). An extended definition of coherent risk measures in general probability spaces can be found in Delbaen (2002).

Let us represent a firm $F$ defined by the pair of variables $(X, u)$, where $X=\left(X_{1}, \ldots, X_{n}\right) \in \mathfrak{X}^{n}$ and $u=$ $\left(u_{1}, \ldots, u_{n}\right) \in \mathbb{R}^{n}$ are referred to as the portfolio base and portfolio respectively. $X_{k}$ can be interpreted as the payoff per unit of an asset of type $k \in N=\{1, \ldots, n\}$, and $u_{k}$ as the number of units held for that type of 
asset.

In practical applications, it is obvious that a group of instruments with almost surely identical future net worths should be modeled more simply as a single asset having the corresponding future net worth distribution. This could be done for a set of shares in a certain stock, for example, but generally not for a set of contracts within an insurance segment. The latter case could be represented either by a single asset having the future net worth distribution of the entire segment, or by a separate asset and future net worth distribution for each contract. In the second approach, as will shortly become clear, risk capital will not only be allocated to the segment but also to each individual contract. In contrast with security portfolios, the assets of an insurance portfolio are typically considered individually.

The future period net worth, or payoff, of firm $F$ is equal to $X(u)=\sum_{k \in N} u_{k} X_{k}$. The risk capital $\rho(X(u))$ of firm $F$ is assumed to depend on the payoff, but has to be fully allocated to the portfolio positions $u_{1} X_{1}, \ldots, u_{n} X_{n}$ in the current period. The rule for applying risk capital is called an allocation principle, and is defined as follows.

Definition 2.2 Let the set of firms $\mathrm{F}$ be defined as a set of pairs $(X, u)$, with $X \in \mathfrak{X}^{n}, u \in \mathbb{R}^{n}$, and $n \in \mathbb{N}_{+}=$ $\mathbb{N} \backslash\{0\}$. Given a risk measure $\rho$, an allocation principle on $\mathrm{F}$ is defined as a mapping $A^{\rho}: \mathrm{F} \rightarrow \mathbb{R}^{\mathrm{n}}$

$$
A^{\rho}:(X, u) \mapsto\left[\begin{array}{c}
A_{1}^{\rho}(X, u) \\
\vdots \\
A_{n}^{\rho}(X, u)
\end{array}\right] \quad \text { such that } \sum_{k \in N} A_{k}^{\rho}(X, u)=\rho(X(u)) .
$$

The expression $A_{k}^{\rho}(X, u) / u_{k}$ is called the per-unit risk contribution of position $k$.

Denault (2001) has introduced the following set of axioms, which are claimed to be "necessary properties of a 'reasonable' allocation principle".

Definition 2.3 An allocation principle $A^{\rho}$ on $\mathrm{F}$ is called coherent if the following three properties hold for all $F=(X, u) \in \mathrm{F}:$

1. No undercut: for all $M \subseteq N$, we have $\sum_{k \in M} A_{k}^{\rho}(X, u) \leq \rho\left(\sum_{k \in M} u_{k} X_{k}\right)$.

2. Symmetry: If by joining the subsets $M \subseteq N$ and $\{i, j\}$ the portfolios $i$ and $j$ both make the same contribution to the risk capital; i.e.,

$$
\rho\left(\sum_{k \in M} u_{k} X_{k}+u_{i} X_{i}\right)-\rho\left(\sum_{k \in M} u_{k} X_{k}\right)=\rho\left(\sum_{k \in M} u_{k} X_{k}+u_{j} X_{j}\right)-\rho\left(\sum_{k \in M} u_{k} X_{k}\right),
$$

for all subsets $M \subseteq N$ and $\{i, j\}$, then $A_{i}^{\rho}(X, u)=A_{j}^{\rho}(X, u)$.

3. Riskless allocation: if $X_{n}$ is a riskless instrument with worth 1 , we have $A_{n}^{\rho}(X, u)=\rho\left(u_{n}\right)=-u_{n}$.

Compared to the original version presented by Denault, three modifications have been made. First, for reasons 
explained below, $\mathrm{F}$ is introduced to control the set of firms considered. ${ }^{1}$ Choosing $\mathrm{F}=\mathrm{F}_{\text {all }}$ with $\mathrm{F}_{\text {all }}=$ $\left\{(X, u) \mid X \in \mathfrak{X}^{n}, u \in \mathbb{R}^{n}\right\}$ recovers Denault's original (2001) axiomatics. Second, the symmetry property is defined formally as well as verbally. This is because various mathematical interpretations of this criterion can be found in the literature (cf. Albrecht, 2004 and Hürlimann, 2001). Third, the property of riskless allocation is stated slightly differently. It is easily seen, however, that for $F=F_{\text {all }}$ both definitions of a coherent capital allocation principle are equivalent.

Denault (2001), Tasche (2000), and Kalkbrener (2005) propose using the marginal risk to set the per-unit risk contribution. In order to introduce the resulting capital allocation principle formally, we define the function $\rho_{X}: \mathbb{R}^{n} \rightarrow \mathbb{R}$ by $\rho_{X}: u \mapsto \rho(X(u))$ for ease of notation. $\rho_{X}$ therefore represents the risk associated with portfolio $u$ given portfolio base $X$. Moreover, for a given risk measure $\rho$ and portfolio base $X$, we let $\mathrm{F}_{X}^{\rho}=$ $\{(X, u) \mid u \in U\}$ where $U$ satisfies the following three conditions. First, $U$ is a cone, i.e., for all $u \in U$ and $\lambda>0$ we also have $\lambda u \in U$. Second, $\rho_{X}$ is differentiable at every $u \in U$. Third, $\rho_{X}$ is positively homogeneous on $U$, i.e., $\rho_{X}(\lambda u)=\lambda \rho_{X}(u)$ for all $\lambda>0$ and $u \in U$. Obviously, without further conditions, $\mathrm{F}_{X}^{\rho}$ is not uniquely determined in general.

Definition 2.4 For a given risk measure $\rho$ and portfolio base $X$, the mapping $A_{\nabla}^{\rho}: \mathrm{F}_{X}^{\rho} \rightarrow \mathbb{R}^{n}$ defined as $A_{\nabla}^{\rho}$ : $(X, u) \mapsto A_{\nabla}^{\rho}(X, u)=u * \nabla \rho_{X}(u)$ is called the gradient allocation principle associated with $\rho$ on $\mathrm{F}_{X}^{\rho}$. Here * denotes the Hadamard or component-wise product, so $A_{\nabla}^{\rho}(X, u)$ is a vector.

Note that under the three assumptions imposed on $\mathrm{F}_{X}^{\rho}$, Euler's theorem yields $u \cdot \nabla \rho_{X}(u)=\rho_{X}(u)$, i.e., an allocation of the entire risk capital over all $u \in U$. In particular, this means that the gradient allocation principle is actually an allocation principle on $\mathrm{F}_{X}^{\rho}$. The gradient allocation principle is sometimes referred to in the literature as the Euler principle (cf. Albrecht, 2004 and Tasche, 2005) or the Myers \& Read method (cf. Urban et al., 2004). As pointed out by Denault (2001), the gradient coincides with the Aumann-Shapley value if the risk measure is positively homogeneous.

The allocation principle introduced here is based on the gradient of $\rho_{X}$. Its applicability to arbitrary firms, i.e., to all $F \in \mathrm{F}$, clearly presumes that $\rho_{X}$ is differentiable at $u$ for all $X \in \mathfrak{X}^{n}$ and all $u \in \mathbb{R}^{n}$. A necessary and sufficient condition for this level of generality is the Gâteaux-differentiability of $\rho$ on $\mathfrak{X}$. As pointed out by Fischer (2003), however, this property is not desirable since it is equivalent to the linearity of $\rho$ provided that $\rho$ is subadditive and positively homogeneous. A linear risk measure does not account for diversification effects, in the sense that the total risk of a portfolio is equal to the sum of the risks associated with each subportfolio,

\footnotetext{
${ }^{1}$ In Denault (2001), results concerning the coherence of the fuzzy value given by the gradient with respect to a portfolio $u$ are stated under the assumption that the cost function is differentiable at $u$ for a given portfolio base $X$. Since coherent fuzzy values are associated with coherent allocation principles, this result suggests the incorporation of $\mathrm{F}$ into the original definition.
} 
i.e., that $\rho_{X}(u)=\sum_{k \in N} \rho_{X}\left(u_{k} \cdot e_{k}\right)$ where $e_{k}$ is the $k$ th canonical unit vector in $\mathbb{R}^{n}$.

Two findings can be drawn from this discussion. The first concerns the proper definition of coherence with respect to an allocation principle. Choosing $F=F_{\text {all }}$ in Definition 2.3 implicitly requires the Gâteauxdifferentiability of $\rho$ on $\mathfrak{X}$, i.e., the linearity of $\rho$. In this case, the gradient allocation principle is clearly coherent. The introduction of $\mathrm{F}$ is therefore motivated by the desire to maintain the significance of coherence for non-linear risk measures by generalizing the original definition of the concept. The second conclusion is that imposing weaker differentiability requirements is a reasonable approach. In section 3.1, useful statements regarding the properties of the gradient allocation principle given a portfolio base $X$ and risk measure $\rho$ will be derived. To ensure that the gradient allocation principle $A_{\nabla}^{\rho}$ is well-defined, the set of firms considered is taken to be $\mathrm{F}_{X}^{\rho}$. Section 3.2, on the other hand, investigates which properties of the risk measure are implied by certain attributes of the gradient allocation principle. The results of this section presume that $\rho$ is Gâteaux-differentiable on $V_{-1}=V \backslash\langle 1\rangle$, where $V$ is a vector subspace of $\mathfrak{X}$ over $\mathbb{R}$ and $\langle 1\rangle$ is the linear span of a random variable that is equal to 1 a.s. The mathematical reasoning for this constraint is as follows: first, the Gâteaux-differentiability of $\rho$ at a constant random variable implies the Gâteaux-differentiability of $\rho$ at 0 , if $\rho$ is translation invariant; and second, the result that the Gâteaux-differentiability of $\rho$ on $\mathfrak{X}$ is a sufficient condition for its linearity depends on the Gâteaux-differentiability of $\rho$ at 0 . Assuming the Gâteauxdifferentiability of $\rho$ on $V_{-1}$ therefore ensures that the gradient allocation principle will apply to all firms within the set $\mathrm{F}_{V}=\left\{(X, u) \mid X \in V^{n}, n \in \mathbb{N}_{+}, X(u) \in V_{-1}\right\}$. Thus, the only firms excluded are those that have a known, constant future payoff. From the economic point of view, these firms can be considered irrelevant. Let us note that $V=\mathfrak{X}$ in many practical applications. The set of differentiable risk measures on $\mathfrak{X}_{-1}$ is a proper superset of the set of all linear risk measures. Two of the three most commonly used risk measures presented in section 4 , for example, are Gâteaux-differentiable on $\mathfrak{X}_{-1}$ but non-linear. The results derived in the next section reveal that their corresponding gradient allocation principles are not coherent.

\section{Links between the two coherence concepts}

Throughout this section we consider both coherence concepts: the coherence of the risk measure used, and the coherence of the gradient allocation principle derived from the risk measure. Various results connecting the two concepts are presented. In section 3.1 it is shown that certain of the four axioms given in Definition 2.1 are sufficient for two of the three axioms given in Definition 2.3. Section 3.2, on the other hand, examines the implications of adopting particular subsets of the coherent gradient allocation principle axioms. 


\subsection{Implications of the coherent risk measure axioms}

Recall that the first link between the two coherence concepts was described along with Definition 2.4: positive homogeneity of the risk measure implies full allocation of the risk measure, under suitable technical assumptions. It will now be shown that for a positively homogeneous risk measure, the property of subadditivity is sufficient for the corresponding gradient allocation principle to satisfy the "no undercut" property.

Theorem 3.1 Let $\rho$ be a risk measure and $X$ a portfolio base. If $\rho$ is subadditive, its gradient allocation principle has the "no undercut" property for all $F \in \mathrm{F}_{X}^{\rho}$.

Proof: Let $\rho$ be subadditive, and $M \subseteq N$. In the case $\mathrm{F}_{X}^{\rho} \neq \emptyset$, consider $(X, u) \in \mathrm{F}_{X}^{\rho}$. By applying Theorem 38 (p. 51) and Theorem 41 (p. 53) from Bullen et al. (2003) to $\rho_{X}$, we can derive the result $\rho_{X}(w) \geq w \cdot \nabla \rho_{X}(v)$ for all $w, v \in U$. The latter inequality proves the "no undercut" property for $v=u$ and $w=\left(w_{1}, \ldots, w_{n}\right)$, where $w_{k}=u_{k}$ if $k \in M$ and $w_{k}=0$ otherwise.

Moreover, it can be easily shown that translation invariance of the risk measure implies that translation invariance of the risk measure implies the riskless allocation property.

Theorem 3.2 Let $\rho$ be a risk measure and $X \in \mathfrak{X}^{n-1} \times\{1\}$ a portfolio base. If $\rho$ is translation invariant, the gradient allocation principle fulfills the riskless allocation property for all $F \in \mathrm{F}_{X}^{\rho}$.

Proof: In the case $\mathrm{F}_{X}^{\rho} \neq \emptyset$, let $(X, u) \in \mathrm{F}_{X}^{\rho}$. Translation invariance of $\rho$ gives $\rho\left(\sum_{k=1}^{n-1} u_{k} X_{k}+u_{n}\right)=\rho\left(\sum_{k=1}^{n-1} u_{k} X_{k}\right)-$ $u_{n}$. Taking the derivative with respect to $u_{n}$ and multiplying by $u_{n}$ shows that $A_{\nabla, n}^{\rho}(X, u)=u_{n} \cdot \frac{\partial}{\partial u_{n}} \rho_{X}(u)=-u_{n}$, i.e., the riskless allocation property.

The combination of these two results is summarized below.

Corollary 3.1 Let $\rho$ be a risk measure and $X$ a portfolio base. If $\rho$ is subadditive and translation invariant (e.g., coherent), then the gradient allocation principle fulfills the no undercut and riskless allocation properties for all $F \in \mathrm{F}_{X}^{\rho}$.

As becomes clear in the next section, however, no subset of the axioms given in Definition 2.1 is sufficient for the symmetry axiom of the allocation principle.

\subsection{Implications of the coherent allocation principle axioms}

As is pointed out above, positive homogeneity of the risk measure is a sufficient condition for full allocation of the risk capital given suitable technical conditions. It can also be shown that it is a necessary condition. Throughout the rest of this paper, the symbol $V$ denotes a vector subspace of $\mathfrak{X}$ over $\mathbb{R}$.

Theorem 3.3 Let $\rho$ be a on $V_{-1}$ Gâteaux-differentiable risk measure. If the full allocation property $(u$. 
$\nabla \rho_{X}(u)=\rho_{X}(u)$ ) holds for all firms $F \in \mathrm{F}_{V}$, then the risk measure $\rho$ is positively homogeneous on $V_{-1}$, i.e., $\rho(\lambda Y)=\lambda \rho(Y)$ for all $\lambda>0$ and all $Y \in V_{-1}$.

Proof: Let $Y \in V_{-1}$ and consider the set of single-position firms $\{(Y, u) \mid u \in \mathbb{R} \backslash\{0\}\}$. Trivially, $Y \in V$ and $Y(u)=u Y \in V_{-1}$ for all $u \in \mathbb{R} \backslash\{0\}$. Assuming that the risk capital is fully allocated for all $F \in\{(Y, u) \mid u \in$ $\mathbb{R} \backslash\{0\}\}$ gives $u \cdot \nabla \rho_{Y}(u)=\rho_{Y}(u)$ for all $u \in \mathbb{R} \backslash\{0\}$. The positive homogeneity of $\rho_{Y}$ on $\mathbb{R} \backslash\{0\}$ follows due to Euler's theorem (cf. Fuente, 2000). Since this holds for all $Y \in V_{-1}$, the positive homogeneity of $\rho$ on $V_{-1}$ is proven.

Theorem 3.4 Let $\rho$ be a on $V_{-1}$ Gâteaux-differentiable risk measure. Moreover, assume that the gradient allocation principle $A_{\nabla}^{\rho}$ has the full allocation property for all firms $F \in \mathrm{F}_{V}$. If the "no undercut" property holds for all firms $F \in \mathrm{F}_{V}$, then the risk measure $\rho$ is subadditive on $V_{-1}$, i.e., we have $\rho\left(X_{1}+X_{2}\right) \leq \rho\left(X_{1}\right)+\rho\left(X_{2}\right)$ for all $X_{1}, X_{2} \in V_{-1}$.

Proof: Let $X_{1}, X_{2} \in V_{-1}$ if $V_{-1} \neq \emptyset$. Consider the firm $F=(X, u)$ with $X=\left(X_{1}, X_{2}\right)$ and $u=(1,1)$. Assuming the "no undercut" property gives $A_{\nabla, k}^{\rho}(X, u) \leq \rho\left(X_{k}\right), k=1,2$. Hence, we obtain $\rho\left(X_{1}\right)+\rho\left(X_{2}\right) \geq A_{\nabla, 1}^{\rho}(X, u)+$ $A_{\nabla, 2}^{\rho}(X, u)$. The right-hand side of the latter inequality is equal to $\rho\left(X_{1}+X_{2}\right)$, due to the full allocation property. This shows that $\rho$ is subadditive on $V_{-1}$.

The next result, together with Theorem 3.2, establishes an equivalence between the riskless allocation property of the gradient allocation principle and the translation invariance property of the risk measure.

Theorem 3.5 Let $V$ contain 1 and let $\rho$ be a on $V_{-1}$ Gâteaux-differentiable risk measure. If the riskless allocation property $\left(\partial \rho_{X}(u) / \partial u_{n}=-1\right)$ holds for all firms $F=(X, u)$ with $X \in V^{n-1} \times\{1\}$ and $X(u) \in V_{-1}$, then $\rho$ is translation invariant on $V_{-1}$, i.e., $\rho(Y+\lambda)=\rho(Y)-\lambda$ for all $Y \in V_{-1}$ and all $\lambda \in \mathbb{R}$.

Proof: Assume that $\rho$ is not translation invariant on $V_{-1}$. This implies the existence of a random variable $Y \in V_{-1}$ and a constant $\bar{u}_{n} \in \mathbb{R} \backslash\{0\}$ such that $\rho\left(Y+\bar{u}_{n}\right) \neq \rho(Y)-\bar{u}_{n}$. Define the function $e: \mathbb{R} \rightarrow \mathbb{R}$ by $e: u_{n} \mapsto \rho\left(Y+u_{n}\right)-\rho(Y)+u_{n}$, and note that $e\left(\bar{u}_{n}\right) \neq 0$. Assuming that the riskless allocation property holds for all firms $F=(X, u)$ with $X \in V^{n-1} \times\{1\}$ and $X(u) \in V_{-1}$, and thus in particular for $F=\left((Y, 1),\left(1, u_{n}\right)\right)$, it follows that the derivative of $e$ equals zero for all $u_{n}$. The implied constancy of $e=e\left(u_{n}\right)$ establishes a contradiction, namely the inequality $\rho(Y) \neq \rho(Y)$, which follows by choosing $u_{n}=0$.

The results stated so far reveal close ties between the properties of positive homogeneity and full allocation, subadditivity and "no undercut", and translation invariance and riskless allocation. One might wonder whether any of the axioms given in Definition 2.1 can be identified with the property of symmetry. An answer to this question is given by the next theorem, which is the main result of this paper. The risk measures that lead to a gradient allocation principle having the symmetry property can be characterized by being linear. 
Theorem 3.6 Let $V$ contain 1 and let $\rho$ be a Gâteaux-differentiable risk measure on $V_{-1}$. Moreover, assume that $\rho$ is translation invariant and sublinear on $V$. Then the gradient allocation principle associated with $\rho$ has the property of symmetry for all firms $F \in \mathrm{F}_{V}$ if and only if $\rho$ is linear on $V$.

Proof: Let $Y \in V_{-1}$. Additionally, set $N=\{1,2\}$ and define a firm $F=(X, u)$ by setting $X_{1}=-0.5 Y, X_{2}=$ $1.5 Y+\rho(1.5 Y)-\rho\left(X_{1}\right)$, and $u=(1,1)$. The only subset of $N$ not containing 1 and 2 is the empty set. In joining the empty set, both positions 1 and 2 will make the same contribution to the risk capital. This is due to the translation invariance of $\rho$ :

$$
\rho\left(X_{2}\right)=\rho\left(1.5 Y+\rho(1.5 Y)-\rho\left(X_{1}\right)\right)=\rho(1.5 Y)-\rho(1.5 Y)+\rho\left(X_{1}\right)=\rho\left(X_{1}\right) .
$$

The symmetry property requires

$$
\begin{aligned}
\lim _{\mathcal{\varepsilon} \rightarrow 0} \frac{\rho\left(X_{1}+X_{2}+\varepsilon X_{1}\right)-\rho\left(X_{1}+X_{2}\right)}{\varepsilon}=A_{\nabla, 1}^{\rho}(X, u) & =A_{\nabla, 2}^{\rho}(X, u) \\
& =\lim _{\mathcal{E} \rightarrow 0} \frac{\rho\left(X_{1}+X_{2}+\varepsilon X_{2}\right)-\rho\left(X_{1}+X_{2}\right)}{\varepsilon} .
\end{aligned}
$$

Both limits exist thanks to the presumed Gâteaux-differentiability of $\rho$ at $Y$ and the translation invariance of $\rho$, so this condition is equivalent to

$$
\lim _{\varepsilon \rightarrow 0} \frac{\rho\left(X_{1}+X_{2}+\varepsilon X_{1}\right)-\rho\left(X_{1}+X_{2}+\varepsilon X_{2}\right)}{\varepsilon}=0 .
$$

Due to the definition of $X_{1}$ and $X_{2}$, and the translation invariance and positive homogeneity of $\rho$, the numerator can be rewritten for small enough values of $\varepsilon$ as:

$$
(1-0.5 \varepsilon) \rho(Y)-(1+1.5 \varepsilon) \rho(Y)+1.5 \varepsilon \rho(Y)-\varepsilon \rho(-0.5 Y),
$$

or equivalently as

$$
-\varepsilon(0.5 \rho(Y)+\rho(-0.5 Y))
$$

Thus, a necessary and sufficient condition to satisfy (3.1) is that the latter expression is equal to zero. This is the case if and only if $\rho(X)+\rho(-X)=0$.

Hence, the symmetry property requires $\rho(X)+\rho(-X)=0$ for all $X \in V_{-1}$. Because of translation invariance the latter equality is also true for all $Z \in\langle 1\rangle$, and thus on $V$. Since $\rho$ is assumed to be sublinear, this implies that $\rho$ is linear.

Consider an arbitrary firm $F=(X, u)$ such that $X_{i}$ and $X_{j}$ satisfy

$$
\rho\left(\sum_{k \in M} u_{k} X_{k}+u_{i} X_{i}\right)-\rho\left(\sum_{k \in M} u_{k} X_{k}\right)=\rho\left(\sum_{k \in M} u_{k} X_{k}+u_{j} X_{j}\right)-\rho\left(\sum_{k \in M} u_{k} X_{k}\right)
$$


for all subsets $M \subseteq N \backslash\{i, j\}$. Note that choosing $M=\emptyset$ reduces this condition to $\rho\left(u_{i} X_{i}\right)=\rho\left(u_{j} X_{j}\right)$. Linearity of the risk measure $\rho$ therefore implies

$$
\lim _{\varepsilon \rightarrow 0} \frac{\left.\rho\left(\sum_{k \in N} u_{k} X_{k}+\varepsilon u_{i} X_{i}\right)-\rho\left(\sum_{k \in N} u_{k} X_{k}+\varepsilon u_{j} X_{j}\right)\right)}{\varepsilon}=\rho\left(u_{i} X_{i}\right)-\rho\left(u_{j} X_{j}\right)=0
$$

and hence $A_{\nabla, i}^{\rho}(X, u)=A_{\nabla, j}^{\rho}(X, u)$.

Remember that linear risk measures do not allow for diversification effects, as was mentioned above. A further discussion of the economically important implications of this last result can be found in the conclusion of this paper. Continuous, linear risk measures are those that can be represented as $\rho(X)=-\mathbb{E}_{\mathbb{Q}_{p}}[X]$, the negative of an expectation taken with respect to a measure $\mathbb{Q}_{\rho}$ on $\Omega$ that is equivalent to $\mathbb{P}$. This representation rephrases Fischer's (2003) Corollary 3.2. Linear risk measures are, of course, coherent. $^{2}$

To clarify the consequences of the theorems derived in this paper, we state the following two corollaries.

Corollary 3.2 Let $V$ contain 1 and let $\rho$ be a Gâteaux-differentiable risk measure on $V_{-1}$. If the gradient allocation principle associated with $\rho$ is coherent on $\mathrm{F}_{V}$, then the risk measure $\rho$ is linear on $V$.

Corollary 3.3 Let $V$ contain 1 and let $\rho$ be a Gâteaux-differentiable risk measure on $V_{-1} \neq \emptyset$. If $\rho$ is nonlinear on $V$, then its gradient allocation principle is not coherent on $F_{V}$.

\section{Applications, and coherent capital allocation in a restricted setting}

\subsection{The gradient allocation principle for some common risk measures}

In this section the definitions of several risk measures are given and results concerning their coherence are summarized. The emerging gradient allocation principles are also stated, and examined with respect to coherence.

We first consider a risk measure which depends on a fixed parameter $a>0$, the mean and standard deviation $\sigma$ of the risky position $X: \rho_{\sigma, a}(X)=-\mathbb{E} X+a \cdot \sigma(X)$. As remarked in Artzner et al. (1999), the risk measure $\rho_{\sigma, a}$ is not coherent. This follows immediately from a result of Delbaen (2002), which states that the axiom " $X \geq 0$ implies $\rho(X) \leq 0$ " can replace the monotonicity axiom in Definition 2.1 without modifying the coherent risk measure axiomatic. Obviously, $\rho_{\sigma, a}$ will fail to meet this condition for a properly chosen $X$. As is shown by Kalkbrener's (2005) Corollary 5.1, the components of the gradient allocation principle for the risk measure $\rho_{\sigma, a}$ are

$$
A_{\nabla, k}^{\rho_{\sigma, a}}(X, u)=-\mathbb{E}\left[u_{k} X_{k}\right]+\alpha \cdot \frac{\operatorname{Cov}\left(X(u), u_{k} X_{k}\right)}{\sigma(X(u))}, \quad k \in N
$$

\footnotetext{
${ }^{2}$ This follows immediately from the representation of coherent risk measures given in Artzner et al. (1999), for example.
} 
provided $\sigma(X(u))>0$. This reveals in particular that the Gâteaux-gradient of $\rho_{\sigma, a}$ exists on $\mathfrak{X}_{-1}$. This principle is called the covariance principle, which is commonly used in practice. The results derived in the last section reveal the following relationship:

Corollary 4.1 Let $V$ contain 1 . The covariance principle fulfills the "no undercut" and riskless allocation properties but not the symmetry property for all $F \in \mathrm{F}_{V}$, and thus is not a coherent capital allocation principle on $\mathrm{F}_{V}$.

Note that in particular, $V=\mathfrak{X}$ may be chosen.

If one understands risk as an asymmetric concept related to outcomes below a certain target, then a risk measure based on the standard deviation is inadequate because both positive and negative deviations from the mean increase risk. Bearing this in mind, the next risk measure we want to consider is $\rho_{\sigma_{-}, a}(Z)=-\mathbb{E} Z+a \cdot \sigma_{-}(Z)$ where $\sigma_{-}(Z)=\sigma\left((Z-\mathbb{E} Z) \cdot \mathbb{1}_{\{Z \leq \mathbb{E} Z\}}\right)$, and $\mathbb{1}_{B}$ denotes the indicator function of $B$. The risk measure $\rho_{\sigma, a}$ is coherent if $0 \leq a \leq 1$, as shown in Fischer (2003), Lemma 4.1. ${ }^{3}$ Applying Fischer's (2003) Proposition 4.8 to the present case, it follows that the components of the gradient allocation principle for the risk measure $\rho_{\sigma_{-}, a}$ exist on $\mathfrak{X}_{-1}$ and are given by

$$
A_{\nabla, k}^{\rho_{\sigma_{-}, a}}(X, u)=-\mathbb{E}\left[u_{k} X_{k}\right]+\alpha \cdot \frac{\operatorname{Cov}_{-}\left(X(u), u_{k} X_{k}\right)}{\sigma_{-}(X(u))}, \quad k \in N .
$$

This allocation principle is called the semi-covariance principle. A result analogous to Corollary 4.1 can be stated:

Corollary 4.2 Let V contain 1 . The semi-covariance principle fulfills the "no undercut" and riskless allocation properties but not the symmetry property for all $F \in \mathrm{F}_{V}$, and thus is not a coherent capital allocation principle on $\mathrm{F}_{V}$.

Due to regularity requirements, one risk measure that plays an important role in practical applications is $\rho_{\operatorname{VaR}_{\alpha}}(X)=-q_{\alpha}(X)$, where $\alpha \in(0,1)$ and $q_{\alpha}(X)=\inf \{x \mid \mathbb{P}(X \geq x) \geq \alpha\}$ denotes the upper $\alpha$-quantile of $X$. The measure $\rho_{\mathrm{VaR}_{\alpha}}$ is called the value-at-risk of $X$ at level $\alpha$, and represents the smallest value such that the probability of the future net worth being not smaller this value is at least $\alpha$. The value-at-risk measure is not coherent since it is not subadditive (e.g., Acerbi and Tasche, 2002, Example 2.4). ${ }^{4}$.

The smallest coherent and law-invariant risk measure, which dominates the value-at-risk measure ${ }^{5}$ at level $\alpha$, is $\rho_{E S_{\alpha}}(X)=-(1-\alpha)^{-1}\left(\mathbb{E}\left[X \mathbb{1}_{\left\{X \leq q_{\alpha}(X)\right\}}\right]-q_{\alpha}(X)\left[\alpha-\mathbb{P}\left(X \leq q_{\alpha}(X)\right)\right]\right)$. This is called the expected shortfall

\footnotetext{
${ }^{3}$ Subadditivity holds for all $a>0$. Artzner et al. (1999) remark that $\rho_{\sigma_{-}, a}$, on the other hand, is not subadditive.

${ }^{4}$ Results concerning the existence and computability of the partial derivatives of $\rho_{\mathrm{VaR}_{\alpha}}$ can be found in Gouriéroux et al. (2000) and Tasche (2000).

${ }^{5}$ See Acerbi and Tasche (2002) and Delbaen (2002) for details.
} 
at level $\alpha$, and measures the expected loss under the hypothesis that the future net worth is below the specified upper $\alpha$-quantile. In Acerbi and Tasche (2002), Proposition 3.1 shows that $\rho_{E S_{\alpha}}$ is a coherent risk measure. Furthermore, Lemma 5.6 from Tasche (2000) implies that the components of its associated gradient allocation principle exist provided that $X(u)$ is continuous, under suitable assumptions. They are given by

$$
A_{\nabla, k}^{\rho_{E S_{\alpha}}}(X, u)=-\mathbb{E}\left[u_{k} X_{k} \mid X(u) \leq q_{\alpha}(X(u))\right], \quad k \in N
$$

This allocation principle is called the expected shortfall principle. If $V$ contains 1 and $\rho_{E S_{\alpha}}$ is Gâteauxdifferentiable on $V_{-1}$, however, Theorem 3.6 shows that the expected shortfall principle is not coherent on $F_{V}$ due to the non-linearity of $\rho_{E S_{\alpha}}{ }^{6}$

\subsection{A coherent gradient allocation principle for centered random variables}

Theorem 3.6 shows that symmetry of the allocation principle is equivalent to linearity of the risk measure under suitable assumptions. In Urban et al. (2004) these considerations are restricted to the space $\mathfrak{X}_{\mathrm{cn}} \subset \mathfrak{X}$, which consists of all real-valued, centered random variables. This restriction can be justified, for example, if one understands $S_{1}, \ldots, S_{n}$ as the different risk claims of an insurance portfolio and assumes premiums equal to $\mathbb{E} S_{1}, \ldots, \mathbb{E} S_{n}$. Then the future income for claim $k$ is the centered random variable $X_{k}=\mathbb{E} S_{k}-S_{k}$, on which the capital allocation can be based (cf. Urban et al., 2004). The translation invariance of the risk measure and the riskless property of the allocation principle are thus trivially satisfied. In this restricted framework the covariance principle also has all the remaining coherence properties, in particular the symmetry property.

Theorem 4.1 The covariance principle is coherent on the set $\mathrm{F}_{c n}=\left\{(X, u) \mid X \in \mathfrak{X}_{c n}^{n}, n \in \mathbb{N}_{+}, X(u) \in \mathfrak{X}_{-1}\right\}$. Proof: Let $F=(X, u) \in \mathrm{F}_{\mathrm{cn}}$. Because the "no undercut" property of the covariance principle is already stated in Corollary 4.1, the symmetry property remains to be shown. Let risks $X_{i}$ and $X_{j}$ both make the same contribution to the risk capital by joining any subset $M \subseteq N \backslash\{i, j\}$. Choosing $M=\emptyset$ and $M=N \backslash\{i, j\}$ yields

$$
\sigma^{2}\left(u_{i} X_{i}\right)=\sigma^{2}\left(u_{j} X_{j}\right)
$$

and

$$
\sigma^{2}\left(\sum_{k \in N \backslash\{j\}} u_{k} X_{k}\right)=\sigma^{2}\left(\sum_{k \in N \backslash\{i\}} u_{k} X_{k}\right),
$$

or equivalently,

$$
\sigma^{2}\left(u_{i} X_{i}\right)+2 \cdot \operatorname{Cov}\left(\sum_{k \in N \backslash\{i, j\}} u_{k} X_{k}, u_{i} X_{i}\right)=\sigma^{2}\left(u_{j} X_{j}\right)+2 \cdot \operatorname{Cov}\left(\sum_{k \in N \backslash\{i, j\}} u_{k} X_{k}, u_{j} X_{j}\right) .
$$

\footnotetext{
${ }^{6}$ Denault (2001), however, insinuates that the contrary holds.
} 
Combining equations (4.1) and (4.2) gives

$$
\operatorname{Cov}\left(\sum_{k \in N \backslash\{i, j\}} u_{k} X_{k}, u_{i} X_{i}\right)=\operatorname{Cov}\left(\sum_{k \in N \backslash\{i, j\}} u_{k} X_{k}, u_{j} X_{j}\right) .
$$

Using the latter equation with (4.1) shows that

$$
\begin{aligned}
A_{\nabla, i}^{\rho_{\sigma, a}}(X, u) & =\frac{a}{\sigma(X(u)} \cdot\left(\sigma^{2}\left(u_{i} X_{i}\right)+\operatorname{Cov}\left(u_{j} X_{j}, u_{i} X_{i}\right)+\operatorname{Cov}\left(\sum_{k \in N \backslash\{i, j\}} u_{k} X_{k}, u_{i} X_{i}\right)\right) \\
& =\frac{a}{\sigma(X(u)} \cdot\left(\sigma^{2}\left(u_{j} X_{j}\right)+\operatorname{Cov}\left(u_{i} X_{i}, u_{j} X_{j}\right)+\operatorname{Cov}\left(\sum_{k \in N \backslash\{i, j\}} u_{k} X_{k}, u_{j} X_{j}\right)\right) \\
& =A_{\nabla, j}^{\rho_{\sigma, a}}(X, u)
\end{aligned}
$$

due to the symmetry of the covariance operator, which proves the symmetry of the covariance principle.

Note that the "no undercut property" of the covariance principle is equivalent to the condition that the correlation coefficient between $\sum_{k \in M} X_{k}$ and $\sum_{k \in N} X_{k}$ does not exceed 1.

The following two examples show that the semi-covariance and expected shortfall principles do not fulfill the symmetry property on $\mathrm{F}_{\mathrm{cn}}$, and thus are not coherent on $\mathrm{F}_{\mathrm{cn}}$. In both cases, we let $F=(X, u)$ with $X=\left(X_{1}, X_{2}\right)$ and $u=(1,1)$. By having $\rho\left(X_{1}\right)=\rho\left(X_{2}\right)$, but $A_{\nabla, 1}^{\rho}(X, u) \neq A_{\nabla, 2}^{\rho}(X, u)$, we can show the failure of symmetry.

Example 1 First we suppose $\rho=\rho_{\sigma_{-}, a}$ and $\Omega=\left\{\omega_{1}, \omega_{2}, \omega_{3}\right\}$, with $\mathbb{P}\left(\omega_{1}\right)=0.2$ and $\mathbb{P}\left(\omega_{2}\right)=\mathbb{P}\left(\omega_{3}\right)=0.4$. Moreover, let $X_{1}$ and $X_{2}$ be the centered random variables defined by

$$
X_{1}(\omega)=\left\{\begin{aligned}
-6 & \text { if } \quad \omega=\omega_{1}, \\
-1 & \text { if } \quad \omega=\omega_{2}, \\
4 & \text { if } \quad \omega=\omega_{3},
\end{aligned} \quad \text { and } \quad X_{2}(\omega)=\left\{\begin{array}{rll}
2 \cdot \sqrt{10}+6 & \text { if } & \omega=\omega_{1}, \\
-\sqrt{10} & \text { if } & \omega=\omega_{2}, \\
-3 & \text { if } & \omega=\omega_{3} .
\end{array}\right.\right.
$$

This gives $\rho_{\sigma_{-}, a}\left(X_{1}\right)=a \cdot \sqrt{7.6}=\rho_{\sigma_{-}, a}\left(X_{2}\right)$, but different amounts are allocated according to the semi-covariance principle: $A_{\nabla, 1}^{\rho_{\sigma_{-}, a}}(X, u)=\sqrt{0.4} \cdot a$,

Example 2 Now set $\rho=\rho_{E S_{8 / 9}}$, and let $X_{1}$ and $X_{2}$ be independent with densities $f_{1}(x)=1 / 3 \cdot \mathbb{1}_{[-1.5,1.5]}(x)$ and $f_{2}(x)=2 \cdot(x-2) / 9 \cdot \mathbb{1}_{[-2,1]}(x)$. We have $\mathbb{E} X_{1}=0=\mathbb{E} X_{2}$, and $\rho_{E S_{8 / 9}}\left(X_{1}\right)=\rho_{E S_{8 / 9}}\left(X_{2}\right)=4 / 3$. The quantile of $X(u)$ at level $8 / 9$ is $q \equiv q_{8 / 9}(X(u))=9^{1 / 3}-7 / 3$. The vector $\left(X_{1}, X_{2}\right)$ satisfies the differentiability assumptions given by Tasche (2000). The amounts allocated according to the expected shortfall principle are $A_{\nabla, 1}^{\rho_{E S_{8 / 9}}}(X, u)=1715 / 576+49 / 36 \cdot q-7 / 24 \cdot q^{2}-2 / 9 \cdot q^{3}-1 / 36 \cdot q^{4}=.9799790436$ and $A_{\nabla, 2}^{\rho_{E S_{8 / 9}}}(X, u)=$ $343 / 288-49 / 36 \cdot q-7 / 4 \cdot q^{2}-5 / 9 \cdot q^{3}-1 / 18 \cdot q^{4}=.9599580882$. 


\section{Conclusion}

The distribution of risk capital by means of the commonly proposed gradient allocation principle is studied with respect to the axioms defining the coherence of risk measures and allocation principles in general. It is shown that the axiom pairs of positive homogeneity and full allocation, subadditivity and "no undercut", and translation invariance and riskless allocation are equivalent. It is further pointed out that the symmetry property holds if and only if the risk measure is linear.

One reason for the employment of coherent risk measures to determine the overall risk capital is given in Artzner et al.'s (1999) justification of the coherent risk measure axioms. The results presented in this paper constitute a further motivation for three of the four coherent risk measure axioms: the risk measure must be positively homogeneous, subadditive, and translation invariant for the gradient allocation principle to have the properties of full allocation, "no undercut" and riskless allocation respectively.

Postulating a coherent risk measure, whose usage is strongly suggested, we find that the associated gradient allocation principle will have the full allocation, "no undercut" and riskless allocation properties. Monotonicity is not needed to derive the coherent allocation axioms, but is desirable from an economic point of view. Demanding the symmetry property as well comes at the price of linearity in the risk measure, which will then not account for diversification effects. One may conclude that imposing the symmetry axiom is too restrictive, and therefore inappropriate. This finding is in line with the following intuition: since a risk measure expresses the risk of a portfolio as a single number, requiring that contributing to the risk capital by joining subportfolios uniquely determines the allocated amount is a strong constraint. The fact that the gradient allocation principle associated with a coherent risk measure fulfills all the economically significant coherent allocation axioms further justifies the gradient allocation principle.

By using the gradient allocation principle with an appropriate risk measure, the covariance, semi-covariance and expected shortfall principles can all be obtained. Application of the insights gained in this paper reveals that none of these three allocation principles is coherent in general. When restricting to centered random variables, however, the covariance principle is coherent. It is noteworthy that the risk measure leading to the covariance principle is neither linear nor coherent. If adhering to symmetry, the covariance principle is justified as a solution when considering centered random variables. 


\section{References}

Acerbi, C., and D. Tasche, (2002): Expected Shortfall: a natural coherent alternative to Value at Risk, Economic Notes 31(2), 379-388.

Albrecht, P. (2004): Risk Based Capital Allocation, in Encyclopedia of Actuarial Science, Chichester: Wiley.

Artzner, P., F. Delbaen, J. M. Eber, and D. Heath (1997): Thinking Coherently, RISK 10(11), 68-71.

Artzner, P., F. Delbaen, J.-M. Eber, and D. Heath (1999): Coherent Measures of Risk, Mathematical Finance 9(3), 203-228.

Bullen, P. S., D. S. Mitrinovic, and M. Vasic (2003): Handbook of Means and Their Inequalities, Dordrecht, Boston, London: Kluwer.

Delbaen, F. (2002): Coherent Risk Measures on General Probability Spaces. in Advances In Finance and Stochastics - Essays in Honour of Dieter Sondermann, K. Sandmann and P. J. Schönbucher, eds. Berlin: Springer.

Denault, M. (2001): Coherent Allocation of Risk Capital, Journal of Risk 4(1), 7-21.

Fuente, A. de la (2000): Mathematical Methods and Models for Economists, Cambridge: Cambridge University Press.

Gouriéroux, C., J. P. Laurent, O. Scaillet (2000): Sensitivity analysis of values at risk, Journal of Empirical Finance 7(3), 225-245.

Fischer, T. (2002): Risk Capital Allocation by coherent risk measures based on one-sided moments, Insurance: Mathematics and Economics 32(1), 135-146

Hürlimann, W. (2001): Analytical evaluation of economic risk capital and diversification using linear Spearman copulas, Working paper.

Kalkbrener, M. (2005): An Axiomatic Approach to Capital Allocation, Mathematical Finance 15(3), 425437.

Tasche, D. (2000): Risk contributions and performance measurement, Working Paper, TU München.

Tasche, D. (2002): Expected Shortfall and Beyond, Journal of Banking and Finance 26(7), 1519-1533.

Tasche, D. (2005): Risk contributions in an asymptotic multi-factor framework, Working Paper, TU München.

Urban, M., J. Dittrich, C. Klüppelberg, R. Stölting (2004): Allocation of Risk Capital to Insurance Portfolios, Blätter DGVFM 26, 389-406. 